\title{
Rigorous contour integral analysis of generalized Keldysh theory of strong laser photoionization
}

\author{
H. Mineo ${ }^{\text {a,* }}$, S.D. Chao ${ }^{\text {b, }}{ }^{\text {, K. Nagaya }}{ }^{\text {a }}$, K. Mishima ${ }^{\text {c }}$, M. Hayashi ${ }^{d}$, S.H. Lin ${ }^{\text {a }}$ \\ a Institute of Atomic and Molecular Sciences, Academia Sinica, P.O. Box 23-166, Taipei 106, Taiwan, ROC \\ ${ }^{\mathrm{b}}$ Institute of Applied Mechanics, National Taiwan University, Taipei 106, Taiwan, ROC \\ ${ }^{\mathrm{c}}$ CREST, Japan Science and Technology Agency, 4-1-8 Honcho, Kawaguchi-shi, Saitama 332-0012, Japan \\ ${ }^{\mathrm{d}}$ Center for Condensed Matter Sciences, National Taiwan University, Taipei 106, Taiwan, ROC
}

Received 22 November 2006; in final form 17 January 2007

Available online 23 March 2007

\begin{abstract}
Based on the residue theorem we present a rigorous and detailed mathematical treatment for computing a contour integral appearing in the Keldysh theory of the photoionization rate for the hydrogen atom. We consider two different contours in the $u=\sin \theta$ domain and the unique contour in the $z=\exp (\mathrm{i} \theta)$ domain $(\theta=\omega t)$. Particularly in $z=\exp (\mathrm{i} \theta)$ domain we find that one of the poles exists at $z=0$ which corresponds to a large enclosed circle in the $u=\sin \theta$ domain. Consistent results are obtained for both domains. We numerically show that the contribution from $z=0$ is negligibly small compared with that from poles for $\lambda=248 \mathrm{~nm}$.

(C) 2007 Elsevier B.V. All rights reserved.
\end{abstract}

\section{Introduction}

In recent years and previous decades, numerous investigations have been conducted both theoretically and experimentally to understand the interaction of intense laser fields with atoms and molecules [1-4]. Among the theoretical and computational developments and challenges, Keldysh [2] originally formulated the rate constant of a photoionization process, in which ionization takes place from the electronic ground state to a continuum state in the linearly polarized laser field, using a 'length gauge'. $\mathrm{He}$ introduced the adiabaticity parameter, called the Keldysh parameter $\gamma$, that determines whether the process lies in the tunneling or multiphoton region.

The original Keldysh theory was proposed more than 40 years ago. Compared with the KFR theory, however, less

\footnotetext{
* Corresponding author. Fax: +8862 23620200.

E-mail addresses: mineo@gate.sinica.edu.tw (H. Mineo), sdchao@ spring.iam.ntu.edu.tw (S.D. Chao).

${ }^{1}$ Fax: +886 23639290 .
}

theoretical works have been explored. This may be due to the fact that the original Keldysh work assumes highly complicated mathematical treatments [2] that result in complications in dealing with the contour integral appearing in the original Keldysh theory.

Gribakin and Kuchiev [3] were the first to shed light on some of the mathematical details of the original Keldysh theory. Similar to the original Keldysh theory, they have also applied the saddle point method to the time integral in the Keldysh formula. In their approach the integral in the $u=\sin \theta$ domain was transformed to that in the $\theta$ domain $(\theta=\omega t)$. Two saddle points which lie in the lower half plane in the $\theta$ domain were not taken into account because these saddle points are not physically allowed $[3,4]$. Mishima et al. $[5,6]$ have applied the residue theorem to the time integral, and then the time integral obtained was exactly twice of that obtained by the saddle point method [3]. We note that Mishima et al. and Keldysh have not taken into account the pole in the lower half plane.

There are some ambiguities among their results, mathematical approaches and treatments of the residues. Although, the photoionization rate of the hydrogen atom 
agrees qualitatively with the numerical results [7], it is very necessary to clarify those ambiguities when the Keldysh theory is applied to diatomic and polyatomic molecules to compare the theoretical results for photoionization rate with experimental data $[8,9]$.

In the present Letter, we will remove these ambiguities and provide a mathematically rigorous and complete treatment for the time integral by studying the applicability of the residue theorem to Keldysh theory for photoionization rate. For this purpose we will consider the $u=\sin \theta$ and $z=\exp (\mathrm{i} \theta)$ domains to perform contour integrals. In the $u=\sin \theta$ domain we introduce two different contours because in this domain we need to choose an appropriate contour. To further confirm the result we also examine the contour integral in the $z=\exp (\mathrm{i} \theta)$ domain where a contour is automatically given as $|z|=1$. For these different approaches we obtain the same results. Especially in the $z=\exp (\mathrm{i} \theta)$ domain we will show that the two poles in the lower half plane in the $\theta$ domain are automatically excluded. These are omitted by hand in the saddle point method $[3,4]$ because they are not physically allowed. Our mathematical treatment agrees with their physical treatment.

\section{Brief theoretical background of generalized Keldysh theory}

Keldysh's original theory [2,3] has adopted the length gauge, and the ionized electron is described by the Volkov wave function. The photoionization rate, $W_{0}$, of the electron in the ground state of a hydrogen atom, in the presence of the linearly polarized strong laser field $\vec{F} \cos \omega t$, is given by

$$
\begin{aligned}
W_{0}= & \frac{2 \pi}{\hbar} \sum_{n>\left[\left(I_{0}+U_{p}\right) /(\hbar \omega)\right]} \\
& \times \int \frac{\mathrm{d}^{3} p}{(2 \pi \hbar)^{3}}|L(\vec{p})|^{2} \delta\left(\frac{p^{2}}{2 m_{e}}+I_{0}+U-n \hbar \omega\right),
\end{aligned}
$$

where $I_{0}=\hbar^{2} /\left(2 m_{e} a_{0}^{2}\right)$ is the ionization potential and $U=(e F)^{2} /\left(4 m_{e} \omega^{2}\right)$ is the so-called ponderomotive energy. $L(\vec{p})$ is given by the integral over $\theta=\omega t$,

$$
\begin{aligned}
L(\vec{p})= & \frac{1}{2 \pi} \int_{-\pi}^{\pi} \mathrm{d} \theta \cos \theta V_{0}\left(\vec{p}+\frac{e}{\omega} \vec{F} \sin \theta\right) \\
& \times \exp \left(\frac{\mathrm{i}}{\hbar \omega} \int_{0}^{\theta} \mathrm{d} \theta^{\prime}\left[I_{0}+\frac{1}{2 m_{e}}\left(\vec{p}+\frac{e \vec{F}}{\omega} \sin \theta^{\prime}\right)^{2}\right]\right) \\
= & \frac{1}{2 \pi} \int_{-\pi}^{\pi} \mathrm{d} \theta \cos \theta V_{0}\left(\vec{p}+\frac{e}{\omega} \vec{F} \sin \theta\right) \\
& \times \exp \left(\frac{\mathrm{i}}{\hbar \omega}[n \hbar \omega \theta-\xi \cos \theta-U \sin \theta \cos \theta]\right),
\end{aligned}
$$

with $\quad V_{0}(\vec{p})=K_{1 \mathrm{~s}} e p F \cos \theta_{p F}\left(I_{0}+p^{2} /\left(2 m_{e}\right)\right)^{-3}, \quad K_{1 \mathrm{~s}}=$ $-32 i \sqrt{\pi a^{7}} I_{0}^{3} \hbar^{-1}$ and $\xi=e p F \cos \theta_{p F} /\left(m_{e} \omega\right)$, where $\theta_{p F}$ is the angle between $\vec{p}$ and $\vec{F}$, and $n \hbar \omega=I_{0}+U+\frac{p^{2}}{2 m_{e}}$. One of the essential issues in the above formalism is associated with computational methods of the integrals in Eq. (2).

\section{Computation of $L(\vec{p})$ function}

\section{1. $u=\sin \theta$ domain}

Let us compute Eq. (2) by transforming it into a contour integral form in the $u=\sin \theta$ domain. From Eq. (2), $L(\vec{p})$ can be divided into two segments $C_{+}$and $C_{-}$,

$$
\begin{aligned}
L(\vec{p})= & \frac{1}{2 \pi}\left[\int_{-\pi / 2}^{\pi / 2}+\left(\int_{-\pi}^{-\pi / 2}+\int_{\pi / 2}^{\pi}\right)\right] \mathrm{d} \theta \cos \theta V_{0}\left(\vec{p}+\frac{e}{\omega} \vec{F} \sin \theta\right) \\
& \times \exp \left(\frac{\mathrm{i}}{\hbar \omega} j(\sin \theta)\right) \\
= & \frac{1}{2 \pi}\left(\int_{C_{+}}+\int_{C_{-}}\right) \mathrm{d} u V_{0}\left(\vec{p}+\frac{e}{\omega} \vec{F} u\right) \exp \left(\frac{\mathrm{i}}{\hbar \omega} j(u)\right),
\end{aligned}
$$

where $j(u)$ represents an analytical function which will be defined soon later. The integral paths $C_{+}$and $C_{-}$are depicted in Fig. 1, where $-1<u<1$ on the real axis is a cut. Note that the phase of the function $\exp \left(\frac{\mathrm{i}}{\hbar \omega} j(u)\right)$ is different on $C_{+}$and $C_{-}$.

By noting $\cos \theta=+\sqrt{1-u^{2}}(-\pi / 2<\theta<\pi / 2)$ and $\cos \theta=-\sqrt{1-u^{2}}(-\pi \leqslant \theta<-\pi / 2, \pi / 2<\theta<\pi), \quad L(\vec{p})$ can be rewritten as a sum of the integral on each segment,

$$
\begin{aligned}
L(\vec{p})= & \frac{1}{2 \pi} \int_{-1}^{1} \mathrm{~d} u V_{0}\left(\vec{p}+\frac{e}{\omega} \vec{F} u\right) \\
& \times \exp \left(\frac{\mathrm{i}}{\hbar \omega}\left[n \hbar \omega \sin ^{-1} u-\xi \sqrt{1-u^{2}}-U u \sqrt{1-u^{2}}\right]\right) \\
& +\frac{1}{2 \pi} \int_{1}^{-1} \mathrm{~d} u V_{0}\left(\vec{p}+\frac{e}{\omega} \vec{F} u\right) \\
& \times \exp \left(\frac{\mathrm{i}}{\hbar \omega}\left[n \hbar \omega\left(\pi-\sin ^{-1} u\right)+\xi \sqrt{1-u^{2}}+U u \sqrt{1-u^{2}}\right]\right),
\end{aligned}
$$

where the first (second) term gives the contour integral on $C_{+}\left(C_{-}\right)$for $\operatorname{Im} \theta>0$, while on $C_{-}\left(C_{+}\right)$for $\operatorname{Im} \theta<0$.

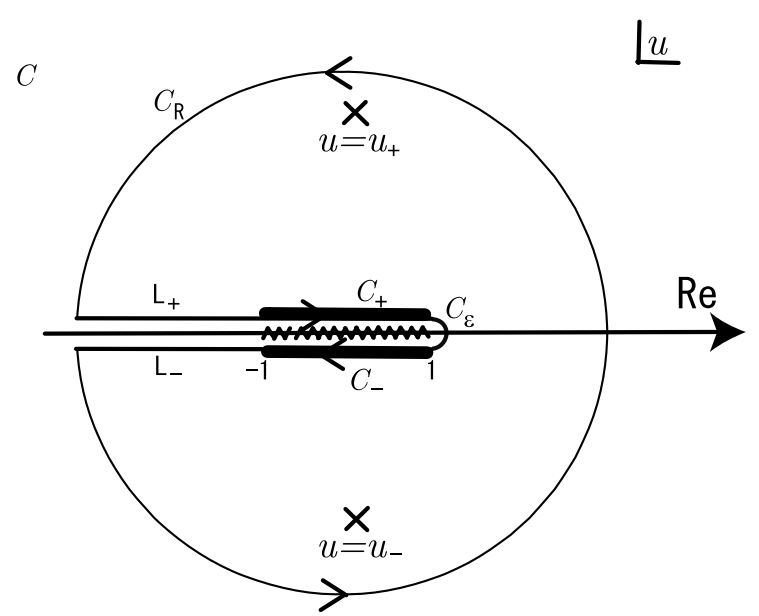

Fig. 1. Contour integral for $L(\vec{p})$ in $u$ domain is depicted. The poles of $V_{0}\left(\vec{p}+\frac{e}{\omega} \vec{F} u\right)$ lie at $u=u_{ \pm}$and there is a cut between $-1<u<1$ on the real axis. 
To compute Eq. (4), we consider the contour integral

$L_{C}(\vec{p})=\frac{1}{2 \pi} \int_{C} \mathrm{~d} u V_{0}\left(\vec{p}+\frac{e}{\omega} \vec{F} u\right) \exp \left(\frac{\mathrm{i}}{\hbar \omega} j(u)\right)$,

where

$$
\begin{aligned}
j(u)= & n \hbar \omega \frac{1}{\mathrm{i}} \log [\mathrm{i} u+(-\mathrm{i} \sqrt{u-1} \sqrt{u+1})] \\
& -\xi(-\mathrm{i} \sqrt{u-1} \sqrt{u+1}) \\
& -U u(-\mathrm{i} \sqrt{u-1} \sqrt{u+1}),
\end{aligned}
$$

and $C$ stands for a contour consisting of the contours $C_{\varepsilon}$, $C_{ \pm}, L_{ \pm}$and $C_{R}$ shown in Fig. 1. In addition, $j(u)$ stands for an analytically continued expression of the function $j(u)$ on $C_{ \pm}$in $\operatorname{Im} \theta>0$, and is defined in the whole $u$ plane so that a residue theorem can be properly applied. We can easily check if Eq. (6) immediately reduces to Eq. (4) by inserting $u=x \pm \mathrm{i} \varepsilon$. We note that if $\operatorname{Im} \theta<0$, an analytically continued expression of the function $j(u)$ is given by

$$
\begin{aligned}
j(u)= & n \hbar \omega \frac{1}{\mathrm{i}} \log [\mathrm{i} u+(+\mathrm{i} \sqrt{u-1} \sqrt{u+1})] \\
& -\xi(+\mathrm{i} \sqrt{u-1} \sqrt{u+1})-U u(+\mathrm{i} \sqrt{u-1} \sqrt{u+1}) .
\end{aligned}
$$

And then the calculated $L_{C}(\vec{p})$ will be mathematically the same as the one obtained in $\operatorname{Im} \theta>0$. However, $L_{C}(\vec{p})$ in $\operatorname{Im} \theta<0$ will not converge for a summation over photon numbers, and in addition they are not physically allowed $[3,4]$. Thus, we only discuss the $\operatorname{Im} \theta>0$ region in the following.

By considering that the contributions from $C_{\varepsilon}$ and $L_{ \pm}$ are zero, we find that $L(\vec{p})$ can be given, in terms of $L_{C}(\vec{p})$ and $L_{R}(\vec{p})$, by

$L(\vec{p})=L_{C}(\vec{p})-L_{R}(\vec{p}) \quad=\sum_{k=+,-} L_{C}(\vec{p}, k)-L_{R}(\vec{p})$,

where

$$
\begin{aligned}
L_{C}(\vec{p}, k)= & 2 \pi \mathrm{i} \operatorname{Res}_{u=u_{k}} \\
& \times \frac{1}{2 \pi} V_{0}\left(\vec{p}+\frac{e}{\omega} \vec{F} u\right) \exp \left(\frac{\mathrm{i}}{\hbar \omega} j(u)\right),
\end{aligned}
$$

and

$L_{R}(\vec{p})=\frac{1}{2 \pi} \int_{C_{R}} \mathrm{~d} u V_{0}\left(\vec{p}+\frac{e}{\omega} \vec{F} u\right) \exp \left(\frac{\mathrm{i}}{\hbar \omega} j(u)\right)$.

The poles at $u=u_{ \pm}$in $L_{C}(\vec{p}, k)$ are the rank-3 poles and given by $u_{ \pm}=\frac{\omega}{e F}\left[-p \cos \theta_{p F} \pm \mathrm{i} \sqrt{2 m_{e} I_{0}+p^{2} \sin ^{2} \theta_{p F}}\right]$. We note that $u_{ \pm}=u_{\mp}^{*}$ and $\operatorname{Im} u_{+}>0, \operatorname{Im} u_{-}<0$.

The term $L_{C}(\vec{p}, k)$ in Eq. (7) is calculated as $L_{C}(\vec{p}, k)$

$$
\begin{aligned}
= & \lim _{u \rightarrow u_{k}} \frac{1}{2 !} \frac{\mathrm{d}^{2}}{\mathrm{~d} u^{2}}\left[\left(u-u_{k}\right)^{3} \frac{1}{2 \pi} V_{0}\left(\vec{p}+\frac{e}{\omega} \vec{F} u\right) \exp \left(\frac{\mathrm{i}}{\hbar \omega} j(u)\right)\right] \\
= & \frac{-K_{1 \mathrm{~s}} e \vec{F} \cdot\left(\vec{p}+\frac{e}{\omega} \vec{F} u_{k}\right) j^{\prime \prime}\left(u_{k}\right)}{16 U_{p}^{3}\left(u_{k}-u_{k}^{*}\right)^{3} \hbar \omega} \\
& \times \exp \left(\frac{\mathrm{i}}{\hbar \omega} j\left(u_{k}\right)\right)(k= \pm)
\end{aligned}
$$

where $j^{\prime}\left(u_{k}\right)=0(k= \pm)$ has been used.
If the argument of $u$ is restricted to $0 \leqslant \operatorname{Arg} u<\pi, j(u)$, $j^{\prime}(u)$ and $j^{\prime \prime}(u)$ are given by

$j(u)=n \hbar \omega \frac{1}{\mathrm{i}} \log \left(i u+\sqrt{1-u^{2}}\right)-\xi \sqrt{1-u^{2}}-U u \sqrt{1-u^{2}}$,

$j^{\prime}(u)=\frac{1}{\sqrt{1-u^{2}}}\left(n \hbar \omega+\xi u+U\left(2 u^{2}-1\right)\right)$,

and

$j^{\prime \prime}(u)=\frac{1}{\sqrt{1-u^{2}}}\left[\xi+4 U_{p} u+\frac{u}{1-u^{2}}\left(n \hbar \omega+\xi u+U\left(2 u^{2}-1\right)\right)\right]$,

respectively. In particular, we obtain for $u=u_{+}$

$j\left(u_{+}\right)=n \hbar \omega \frac{1}{\mathrm{i}} \log \left(i u_{+}+\sqrt{1-u_{+}^{2}}\right)-\xi \sqrt{1-u_{+}^{2}}-U u_{+} \sqrt{1-u_{+}^{2}}$,

$j^{\prime}\left(u_{+}\right)=0$,

and

$j^{\prime \prime}\left(u_{+}\right)=\frac{1}{\sqrt{1-u_{+}^{2}}}\left(\xi+4 U u_{+}\right)=\frac{e \vec{F} \cdot\left(\vec{p}+\frac{e \vec{F}}{\omega} u_{+}\right)}{m_{e} \omega \sqrt{1-u_{+}^{2}}}$.

Note that $j\left(u_{-}\right), j^{\prime}\left(u_{-}\right)$and $j^{\prime \prime}\left(u_{-}\right)$can be easily obtained in a similar fashion (see Appendix A).

By expanding $j^{\prime}(u)$ around $u=u_{ \pm}$, we can derive the relationship between $j^{\prime \prime}\left(u_{ \pm}\right)$and $u_{ \pm}$,

$\sqrt{1-u_{ \pm}^{2}} j^{\prime \prime}\left(u_{ \pm}\right)=2 U\left(u_{+}-u_{-}\right)$.

From Eqs. (10c), (A1) and (11), $L_{C}(\vec{p})$ in Eq. (8) reduces to another expression [6],

$$
\begin{aligned}
L_{C}(\vec{p})= & \sum_{k=+,-} \frac{-m_{e} K_{1 \mathrm{~s}}}{4 U\left(u_{+}-u_{-}\right) \hbar \sqrt{1-u_{k}^{2}}} \\
& \times \exp \left(\frac{\mathrm{i}}{\hbar \omega} j\left(u_{k}\right)\right) .
\end{aligned}
$$

We stress that the residue on the upper half plane gives the same result reported by Mishima et al. [5,6]. But, the residue on the lower half plane is not taken into account in the original Keldysh theory [2] or those by Mishima et al. $[5,6]$. Gribakin and Kuchiev included these two poles and dropped off the other two poles which will not appear in the $u=\sin \theta$ domain but do appear in the $\theta$ domain [3].

Next we consider the circle integral $L_{R}(\vec{p})$ in Eq. (9). For the calculation of $L_{R}(\vec{p})$, we need to expand $V_{0}\left(\vec{p}+\frac{e}{\omega} \vec{F} u\right)$ $\exp \left(\frac{\mathrm{i}}{\hbar \omega} j(u)\right)$ in powers of $u$ and $u^{-1}$, and perform the $\theta$ integral. The results of these expansions are given by

$$
\begin{aligned}
V_{0}\left(\vec{p}+\frac{e}{\omega} \vec{F} u\right)= & \frac{m_{e} \hbar \omega^{2} K_{1 \mathrm{~s}}}{8 U^{3}} \sum_{m=0}^{\infty} C(m) \\
& \times(b+4 c u) u^{-6-m},
\end{aligned}
$$

and 
$\exp \left(\frac{\mathrm{i}}{\hbar \omega} j(u)\right)=\sum_{l, m^{\prime}, j_{1}, j_{2}=0}^{\infty} a_{l}(n) B_{m^{\prime}}(b, c) b_{j_{1}}(b) c_{j_{2}}(c) u^{-n-2 l+m^{\prime}-j_{1}-j_{2}}$,

where $b=\xi /(\hbar \omega)$ and $c=U /(\hbar \omega) . C(m), a_{l}(n), B_{m^{\prime}}(b, c)$, $b_{j_{1}}(b)$ and $c_{j_{2}}(c)$ are given in Appendix B. In Eq. (13b) we have used the fact that the expansion for $\exp (z)$ in $z$ $(=i f(u) /(\hbar \omega))$ converges for arbitrary complex $z$ since its convergence radius is infinity. The aurant expansion for $\exp (z)$ in $u$ will converge because, from Eq. (B4) the positive powers of $u$ which appear in the expansion for $f(u)$ are 'finite', i.e., 2 at most. From Eqs. (7b) and (13), $L_{R}(\vec{p})$ becomes

$$
\begin{aligned}
L_{R}(\vec{p})= & \frac{1}{2 \pi} \int_{C_{R}} \mathrm{~d} u V_{0}\left(\vec{p}+\frac{e}{\omega} \vec{F} u\right) \exp \left(\frac{\mathrm{i}}{\hbar \omega} j(u)\right) \\
= & \frac{1}{2 \pi} \int_{C_{R}} \mathrm{~d} u \frac{m_{e} \hbar \omega^{2} K_{1 \mathrm{~s}}}{8 U^{3}} \\
& \times \sum_{m, l, m^{\prime}, j_{1}, j_{2}=0}^{\infty} C(m) a_{l}(n) B_{m^{\prime}}(b, c) b_{j_{1}}(b) c_{j_{2}}(c) \\
& \times(b+4 c u) u^{M-1},
\end{aligned}
$$

where $M=m^{\prime}-\left(m+5+n+2 l+j_{1}+2 j_{2}\right)$.

By putting $u=R \exp (\mathrm{i} \theta)$ and performing the $\theta$ integral, we obtain

$$
\begin{aligned}
L_{R}(\vec{p})= & \frac{1}{2 \pi} \int_{C_{R}} \mathrm{~d} u V_{0}\left(\vec{p}+\frac{e}{\omega} \vec{F} u\right) \exp \left(\frac{\mathrm{i}}{\hbar \omega} j(u)\right) \\
= & \mathrm{i} \frac{m_{e} \hbar \omega^{2} K_{1 \mathrm{~s}}}{8 U^{3}} \sum_{l, j_{1}, j_{2}, m=0}^{\infty} a_{l}(n) b_{j_{1}}(b) c_{j_{2}}(c) C(m) \\
& \times\left\{b B_{m^{\prime}}(b, c)+4 c B_{m^{\prime}-1}(b, c)\right\},
\end{aligned}
$$

where $m^{\prime}=m+5+n+2 l+j_{1}+2 j_{2}$.

\subsection{Exterior residue method}

The exterior residue theorem [10] assures that the contour on $C^{\prime}$ can be calculated by transforming the contour $C^{\prime}$ to $C_{R^{\prime}}$ as shown in Fig. 2. Thus, Eq. (4) reduces to

$$
\begin{aligned}
L(\vec{p}) & \equiv \frac{1}{2 \pi} \int_{C^{\prime}} \mathrm{d} u V_{0}\left(\vec{p}+\frac{e}{\omega} \vec{F} u\right) \exp \left(\frac{\mathrm{i}}{\hbar \omega} j(u)\right) \\
& =\sum_{k=+,-} L_{C}(\vec{p}, k)-2 \pi i a_{-1},
\end{aligned}
$$

where $\quad a_{-1}=\operatorname{Res}_{u=\infty} \frac{1}{2 \pi} V_{0}\left(\vec{p}+\frac{e}{\omega} \vec{F} u\right) \exp \left(\frac{\mathrm{i}}{\hbar \omega} j(u)\right) \quad$ and $2 \pi i a_{-1}=L_{R}(\vec{p})$. Here, we should emphasize that $a_{-1}$ is the residue of $\frac{1}{2 \pi} V_{0}\left(\vec{p}+\frac{e}{\omega} \vec{F} u\right) \exp \left(\frac{\mathrm{i}}{\hbar \omega} j(u)\right)$ at infinity. In other words, $a_{-1}$ is a coefficient of the $u^{-1}$ term in $\lim _{u \rightarrow \infty} \frac{1}{2 \pi} V_{0}\left(\vec{p}+\frac{e}{\omega} \vec{F} u\right) \exp \left(\frac{\mathrm{i}}{\hbar \omega} j(u)\right)$. Thus, from Eq. (16) We obtain $a_{-1}$ as

$$
\begin{aligned}
a_{-1}= & \frac{1}{2 \pi} \frac{m_{e} \hbar \omega^{2} K_{1 \mathrm{~s}}}{8 U^{3}} \sum_{l, j_{1}, j_{2}, m=0}^{\infty} a_{l}(n) b_{j_{1}}(b) c_{j_{2}}(c) C(m) \\
& \times\left\{b B_{m^{\prime}}(b, c)+4 c B_{m^{\prime}-1}(b, c)\right\}
\end{aligned}
$$

where $M=m^{\prime}-\left(m+5+n+2 l+j_{1}+2 j_{2}\right)$.

It is important to note that the final result for $L(\vec{p})$ is exactly the same as Eqs. (8) and (15). However, the major difference is a choice of the contour adopted for the integrals. More specifically, we need to consider an appropriate contour which can reproduce the original integral in Eq. (4). With the exterior residue method, on the other hand, we just transform the original integral in Eq. (4) into the large circle integral.

\subsection{Residue theorem in $z=\exp (i \theta)$ domain}

Let us now compute a contour integral in $z=\exp (\mathrm{i} \theta)$ domain in which the contour is automatically and uniquely determined as $|z|=1$. In Eq. (2) by replacing $\theta$ with $z=\exp (\mathrm{i} \theta)$, we find

$$
\begin{aligned}
L(\vec{p})= & \frac{1}{2 \pi} \int_{|z|=1} \frac{\mathrm{d} z}{i z}\left(\frac{z+z^{-1}}{2}\right) V_{0} \\
& \times\left(\vec{p}+\frac{e}{\omega} \vec{F} \frac{z-z^{-1}}{2 i}\right) \exp \left(\frac{\mathrm{i}}{\hbar \omega} h(z)\right),
\end{aligned}
$$

with $h(z)=j\left(u=\frac{z-z^{-1}}{2 i}\right)$. In Eq. (18), we can easily see that $V_{0}\left(\vec{p}+\frac{e}{\omega} \vec{F} u\right)$ has rank-3 poles at $z=z_{1}, z=z_{2}, z=-z_{1}^{*}$ and $\quad z=-z_{2}^{*} \quad$ with $\quad z_{1}=i u_{+}+\sqrt{1-u_{+}^{2}} \quad$ and

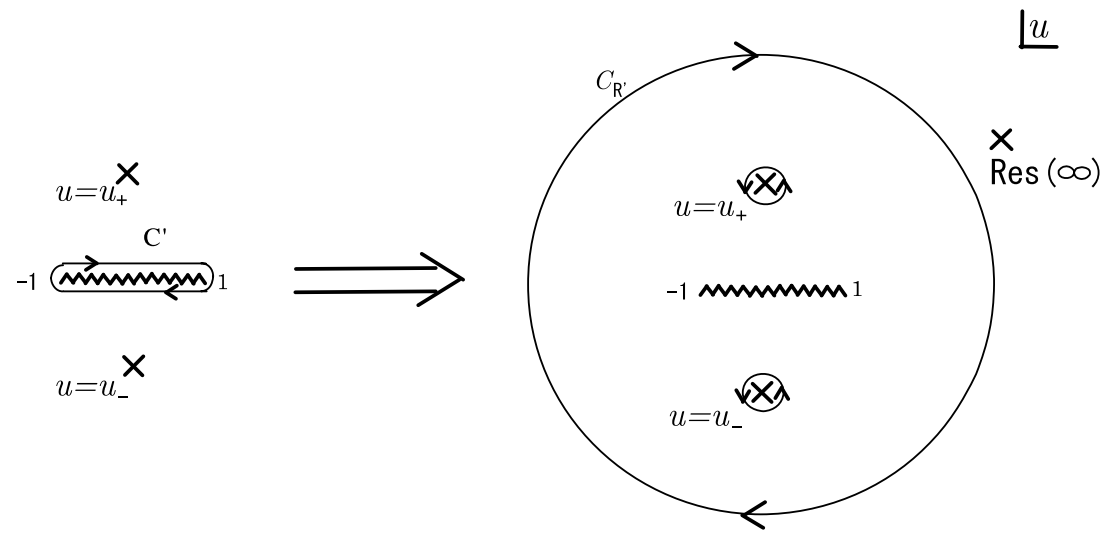

Fig. 2. The contours $C^{\prime}$ and $C_{R^{\prime}}$ are depicted in $u$ domain. On the left hand side the contour integral on $C^{\prime}$ is the original integral in Eq. (7). From left to right, $C^{\prime}$ is transformed to $C_{R^{\prime}}$ which includes the poles at $u=u_{ \pm}$and the residue at infinity. 
$z_{2}=i u_{+}-\sqrt{1-u_{+}^{2}}=-z_{1}^{-1}$. In addition, $\exp \left(\frac{i}{\hbar \omega} h(z)\right)$ has a pole at $z=0$. Thus, the integrand in Eq. (18) has five poles.

By using the relation that $\left|z_{2}\right|>1>\left|z_{1}\right|$ for $0 \leqslant \operatorname{Arg} u_{+}<\pi$, the locations of the poles are schematically shown in Fig. 3. Therefore, $L(\vec{p})$ is given by a sum of the residues at the three poles $z=z_{1}, z=-z_{1}^{*}$, and $z=0$, which are always inside a contour so that we obtain $L(\vec{p})=L_{z=z_{1}}(\vec{p})+L_{z=-z_{1}^{*}}(\vec{p})+L_{z=0}(\vec{p})$ where

$$
\begin{aligned}
L_{z=z_{a}}(\vec{p}) \equiv & 2 \pi \mathrm{i} \operatorname{Res}_{z=z_{a}} \\
& \times \frac{1}{2 \pi} V_{0}\left(\vec{p}+\frac{e}{\omega} \vec{F} \frac{z-z^{-1}}{2 i}\right) \exp \left(\frac{\mathrm{i}}{\hbar \omega} h(z)\right) .
\end{aligned}
$$

We note that each residue at the poles $z=z_{1}, z=-z_{1}^{*}$ and $z=0$ gives the same residue as in Eqs. (8) and (15): $L_{z=z_{1}}(\vec{p})=L_{C}(\vec{p},+), \quad L_{z=-z_{1}^{*}}(\vec{p})=L_{C}(\vec{p},-) \quad$ and $\quad L_{z=0}(\vec{p})=$ $-L_{R}(\vec{p})$.

Fig. 4 shows the poles at $z=z_{1}, z=-z_{1}^{*}, z=z_{2}=-z_{1}^{-1}$, and $z=-z_{2}^{*}=z_{1}^{*-1}$ in the $\theta$ domain. One can also easily see

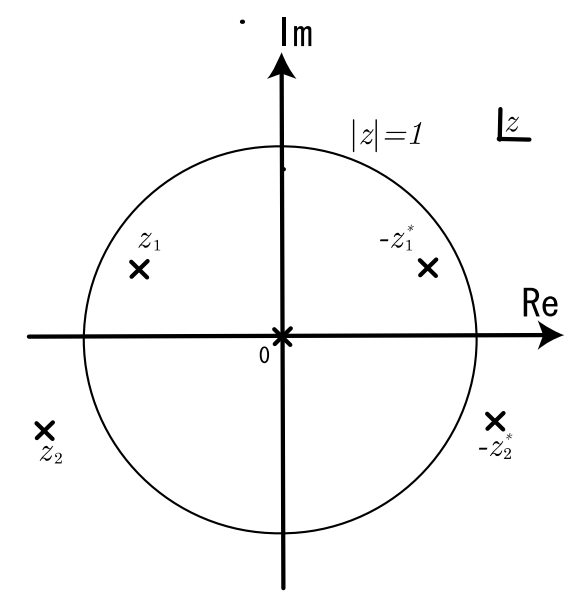

Fig. 3. The contour integral in the $z=\exp (\mathrm{i} \theta)$ domain is depicted. There are five poles lying at $z=z_{1}, z=-z_{1}^{*}, z=z_{2}=-z_{1}^{-1}, z=-z_{2}^{*}=z_{1}^{*-1}$ and $z=0$ with $\left|z_{2}\right|>1>\left|z_{1}\right|$. Three poles $z=z_{1}, z=-z_{1}^{*}$ and $z=0$ lie inside a unit circle. We note that the argument of $z_{1}$ is between $-\pi \leqslant \operatorname{Arg} z_{1}<\pi$.

$\underline{\theta}$

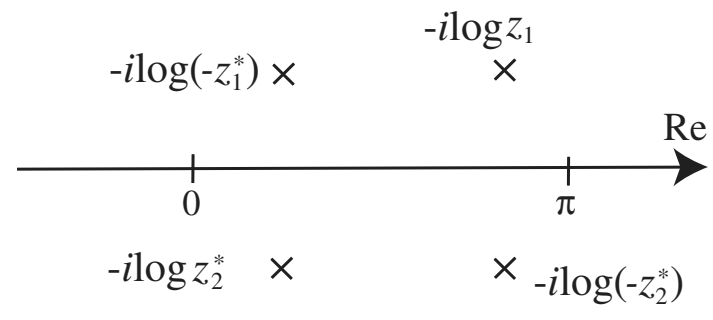

Fig. 4. The poles except $z=0$ in Fig. 3 are depicted in the $\theta$ domain. $\theta=-\mathrm{i} \log z_{1}$ and $\theta=-\mathrm{i} \log \left(-z_{1}^{*}\right)$ always lie in the upper half plane, while $\theta=-i \log z_{2}$ and $\theta=-i \log \left(-z_{2}^{*}\right)$ lie in the lower half plane. There are some relations between $z_{1}$ and $z_{2}, \quad-\pi \leqslant \operatorname{Re}\left(-\mathrm{i} \log z_{1}\right)=$ $\operatorname{Re}\left(-\mathrm{i} \log \left(-z_{2}^{*}\right)\right)<\pi$ and $\operatorname{Re}\left(-\mathrm{i} \log z_{1}\right)+\operatorname{Re}\left(-\mathrm{i} \log \left(-z_{1}^{*}\right)\right)=\pi$. from Fig. 4 that there are two poles in the upper half plane and also two poles in the lower half plane. It is clear that only two poles in the upper half plane can contribute to $L_{C}(\vec{p})$. The importance of the two poles in the upper half plane has also been reported in [3] where the saddle point method was used and the other two poles in the lower half plane were omitted based on physical considerations $[3,4]$. We can assure that our mathematical treatment agrees with their result and physical considerations.

\section{Numerical results and discussions}

Let us compare our results with the literatures $[2,3,5,6]$. Keldysh [2] used the saddle point method and only included $L_{C}(\vec{p},+)$, but his results are different from our results by a factor of 2, i.e. $L_{C}(\vec{p},+)_{\text {Keldysh }}=\frac{1}{2} L_{C}(\vec{p},+)$. This is due to the fact that the saddle point method is an approximated method while the residue theorem is the exact calculation. In the saddle point method, $L_{R}(\vec{p})$ does not appear in the total $L(\vec{p})[2,3,7]$. Mishima et al. [5,6] adopted the residue theorem and obtained $L_{C}(\vec{p},+)$, but he did not include $L_{C}(\vec{p},-)$ and $L_{R}(\vec{p})$. Gribakin and Kuchiev [3] adopted the saddle point method and included $L_{C}(\vec{p},+)$ and $L_{C}(\vec{p},-)$. As in Keldysh's results, their results also differ from ours by a factor of 2 .

It is instructive to compute Eqs. (8) and (15) numerically and compare the photoionization rates $W_{0}$ in Eq. (1), with and without $L_{C}(\vec{p},-)$ and $L_{R}(\vec{p})$. We define for three cases, $L(\vec{p})=L_{C}(\vec{p},+), L(\vec{p})=L_{C}(\vec{p},+)+L_{C}(\vec{p},-)=L_{C}(\vec{p})$, and $L(\vec{p})=L_{C}(\vec{p})-L_{R}(\vec{p})$, as $W_{0}\left(L_{C}(\vec{p},+)\right), \quad W_{0}\left(L_{C}(\vec{p})\right)$, and $W_{0}\left(L_{C}(\vec{p})-L_{R}(\vec{p})\right)$, respectively. They are plotted in Fig. $5 \mathrm{a}$ where the wavelength $\lambda=248 \mathrm{~nm}$ is used. In the actual numerical calculation of $L_{R}(\vec{p})$, we have only added up to 15 instead of $\infty$ for all summations which appear in Eq. (15).

One can easily see that the contribution of $L_{R}(\vec{p})$ is almost negligibly small because the maximum difference between $W_{0}\left(L_{C}(\vec{p})-L_{R}(\vec{p})\right)$ and $W_{0}\left(L_{C}(\vec{p})\right)$ is at most 10 $\%$ as shown in Fig. 5b. Making a comparison between $W_{0}\left(L_{C}(\vec{p},+)\right)$ and $W_{0}\left(L_{C}(\vec{p})\right)$, we find that except for the intensity around $2 \times 10^{14}\left[\mathrm{~W} / \mathrm{cm}^{2}\right]$, the contribution of $L_{C}(\vec{p},-)$ increases the photoionization rate about twofold. The main contribution results from $L_{C}(\vec{p},+)$, while $L_{C}(\vec{p},-)$ is almost the same order of $L_{C}(\vec{p},+)$. Our numerical results suggest that even if $L_{R}(\vec{p})$ is omitted, the qualitative features and the order of magnitude will not change for the 1s hydrogen atom case.

One should use caution here because this fact may not be applicable to other atomic or even molecular systems. Moreover, the numerical stability of $L_{R}(\vec{p})$ should be examined if some extreme conditions are considered. This is partially due to the fact that the structure of $L_{R}(\vec{p})$ may not be suitable for numerical calculations. Replacement of a numerically stable formula for $L_{R}(\vec{p})$ is necessary in future work. One should use extra caution if such systems are studied using the saddle point method or residue theorem. We believe that the validity of applying the residue theorem 

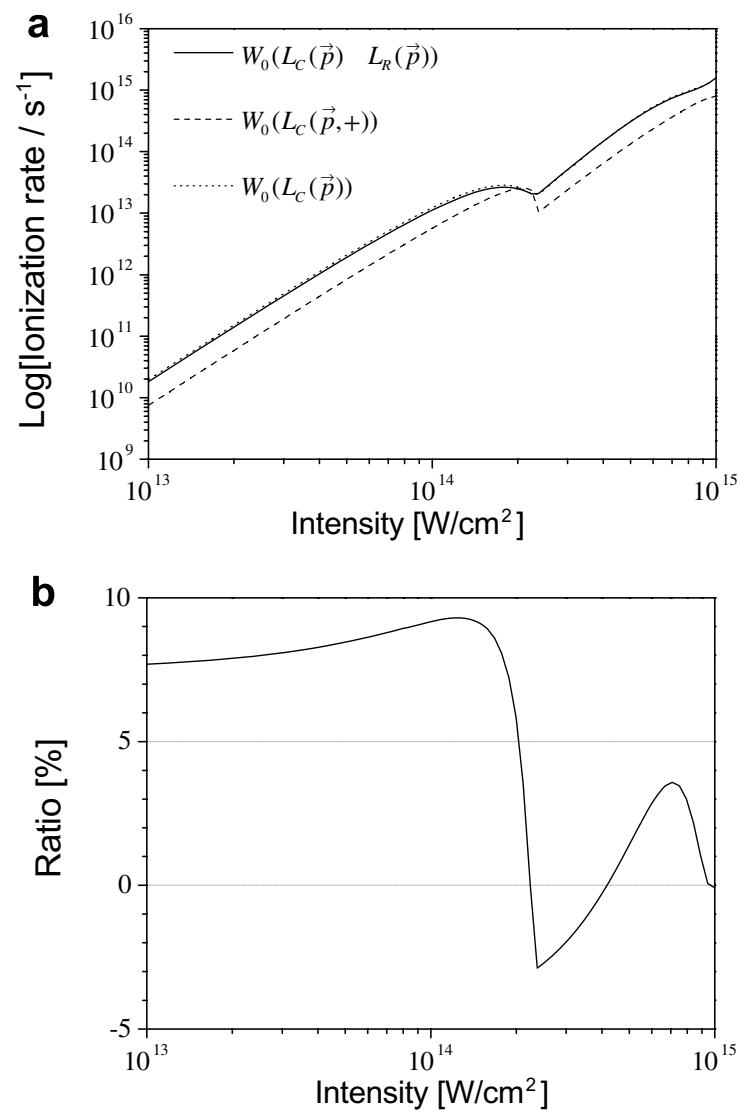

Fig. 5. (a) Photoionization rates for $1 \mathrm{~s}$ hydrogen atom with the wavelength $\lambda=248 \mathrm{~nm}$ are plotted. The solid line stands for $W_{0}\left(L_{C}(\vec{p})-L_{R}(\vec{p})\right)$, the dashed line only includes the contribution from the pole $u=u_{+}$, i.e. $W_{0}\left(L_{C}(\vec{p},+)\right)$ and the dotted line includes the contributions from two poles $u=u_{ \pm}$, i.e. $W_{0}\left(L_{C}(\vec{p})\right)$. (b) Ratio of the photoionization rates $\left[W_{0}\left(L_{C}(\vec{p})-L_{R}(\vec{p})\right)-W_{0}\left(L_{C}(\vec{p})\right)\right] / W_{0}\left(L_{C}(\vec{p})\right)$ are plotted.

to compute the Keldysh's photoionization rate has been proved mathematically without any ambiguity. The contribution from $L_{R}(\vec{p})$ should be examined for other atomic and molecular systems.

\section{Acknowledgements}

This work is supported by National Science council and Academia Sinica.

\section{Appendix A}

$j(u), j^{\prime}(u)$ and $j^{\prime \prime}(u)$ with $u$ restricted to $-\pi \leqslant \operatorname{Arg} u<0$.

If $u$ lies in the lower half plane $-\pi \leqslant \operatorname{Arg} u<0$, we find

$-\mathrm{i} \sqrt{u-1} \sqrt{u+1}=-\sqrt{1-u^{2}}$.

In this case we obtain,

$j(u)=n \hbar \omega \frac{1}{\mathrm{i}}\left[\mathrm{i} \pi-\log \left(i u+\sqrt{1-u^{2}}\right)\right]+\xi \sqrt{1-u^{2}}+U_{p} u \sqrt{1-u^{2}}$,

$j^{\prime}(u)=\frac{-1}{\sqrt{1-u^{2}}}\left(n h \omega+\xi u+U_{p}\left(2 u^{2}-1\right)\right)$, and

$j^{\prime \prime}(u)=\frac{-1}{\sqrt{1-u^{2}}}\left[\xi+4 U_{p} u+\frac{u}{1-u^{2}}\left(n \hbar \omega+\xi u+U_{p}\left(2 u^{2}-1\right)\right)\right]$.

For $u=u_{-}$, we find

$j\left(u_{-}\right)=n \hbar \omega \frac{1}{\mathrm{i}}\left[\mathrm{i} \pi-\log \left(i u_{-}+\sqrt{1-u_{-}^{2}}\right)\right]+\xi \sqrt{1-u_{-}^{2}}+U_{p} u \sqrt{1-u_{-}^{2}}$,

$j^{\prime}\left(u_{-}\right)=0$,

and

$$
\begin{aligned}
j^{\prime \prime}\left(u_{-}\right) & =\frac{-1}{\sqrt{1-u_{-}^{2}}}\left(\xi+4 U_{p} u_{-}\right) \\
& =\frac{-e \vec{F} \cdot\left(\vec{p}+\frac{e \vec{F}}{\omega} u_{-}\right)}{m \omega \sqrt{1-u_{-}^{2}}} .
\end{aligned}
$$

\section{Appendix B}

Results for $C(m), a_{l}(n), B_{m^{\prime}}(b, c), b_{j_{1}}(b)$ and $c_{j_{2}}(c)$.

$C(m), a_{l}(n), B_{m^{\prime}}(b, c), b_{j_{1}}(b)$ and $c_{j_{2}}(c)$ are given as coefficients of Taylor expansions for $V_{0}\left(\vec{p}+\frac{e}{\omega} \vec{F} u\right) \exp \left(\frac{\mathrm{i}}{\hbar \omega} j(u)\right)$. $V_{0}\left(\vec{p}+\frac{e}{\omega} \vec{F} u\right)$ can be expanded by a $u^{-1}$ series as

$V_{0}\left(\vec{p}+\frac{e}{\omega} \vec{F} u\right)=\frac{m_{e} \hbar \omega^{2} K_{1 \mathrm{~s}}}{8 U^{3}} \sum_{m=0}^{\infty} C(m)(b+4 c u) u^{-6-m}$,

where $b=\xi /(\hbar \omega), c=U /(\hbar \omega)$ and

$$
\begin{aligned}
C(m)= & \frac{1}{2(x-y)^{5}}\left[(m+1)(m+2)\left(x^{m+5}-y^{m+5}\right)+2(m+1)\right. \\
& \times(m+5) x y\left(x^{m+3}-y^{m+3}\right) \\
& \left.+(m+4)(m+5) x^{2} y^{2}\left(x^{m+1}-y^{m+1}\right)\right]_{x=u_{+}, y=u_{-}} .
\end{aligned}
$$

In Eq. (7) $\exp \left(\frac{\mathrm{i}}{\hbar \omega} j(u)\right)$ can be rewritten as

$$
\begin{aligned}
\exp \left(\frac{\mathrm{i}}{\hbar \omega} j(u)\right)= & (i u-\mathrm{i} n \sqrt{u-1} \sqrt{u+1})^{n} \\
& \times \exp (-(b+c u) \sqrt{u-1} \sqrt{u+1}),
\end{aligned}
$$

where the first term in Eq. (B2) is

$$
(i u-\mathrm{i} \sqrt{u-1} \sqrt{u+1})^{n}=\sum_{l=0}^{\infty} a_{l}(n) u^{-n-2 l},
$$

with $a_{l}(n)=\left(\frac{\mathrm{i}}{2}\right)^{n} \frac{n_{n+2} C_{l}}{(n+2 l)^{4}} \cdot \exp (-(b+c u) \sqrt{u-1} \sqrt{u+1})$ can be separated into the terms in $u$ and $u^{-1}$ series,

$$
\begin{aligned}
\exp & (-(b+c u) \sqrt{u-1} \sqrt{u+1}) \\
= & \exp \left(-b u-c u^{2}\right) \\
\quad & \times \exp \left(\frac{b u^{-1}}{1+\sqrt{1-u^{-2}}}\right) \exp \left(\frac{c}{1+\sqrt{1-u^{-2}}}\right) .
\end{aligned}
$$

Thus, Taylor expansions for Eq. (B4) are given as follows, 
$\exp \left(-b u-c u^{2}\right)=\sum_{m^{\prime}=0}^{\infty} u^{m^{\prime}} B_{m^{\prime}}(b, c)$,

$\exp \left(\frac{b u^{-1}}{1+\sqrt{1-u^{-2}}}\right)=\sum_{j_{1}=0}^{\infty} u^{-j_{1}} b_{j_{1}}(b)$

$\exp \left(\frac{c}{1+\sqrt{1-u^{-2}}}\right)=\sum_{j_{2}=0}^{\infty} u^{-2 j_{2}} c_{j_{2}}(c)$

where $B_{m^{\prime}}(b, c), b_{j_{1}}(b)$ and $c_{j_{2}}(c)$ are given by

$$
B_{m^{\prime}}(b, c)=(-b)^{m^{\prime}} \sum_{k=0}^{\left[m^{\prime} / 2\right]} \frac{\left(-c / b^{2}\right)^{k}}{k !\left(m^{\prime}-2 k\right) !},
$$

$b_{j_{1}}(b)$

$$
=(b / 2)^{j_{1}}\left(j_{1}-1\right) ! \sum_{k_{1}=0}^{\left[j_{1} / 2\right]} \frac{b^{-2 k_{1}}}{k_{1} !\left(j_{1}-k_{1}\right) !\left(j_{1}-2 k_{1}-1\right) !}
$$

$$
\left(j_{1}>0\right)
$$

$$
\begin{aligned}
& c_{j_{2}}(c) \\
& =2 \exp (c / 2)(c / 8)^{j_{2}}\left(2 j_{2}-1\right) ! \sum_{k_{2}=0}^{j_{2}-1} \frac{(2 / c)^{k_{2}}}{k_{2} !\left(j_{2}-k_{2}-1\right) !\left(2 j_{2}-k_{2}\right) !} \\
& \quad \times\left(j_{2}>0\right),
\end{aligned}
$$

with $b_{0}(b)=1$ and $c_{0}(c)=\exp (c / 2)$.

\section{References}

[1] P. Agostini, L.F. Dimauro, Rep. Prog. Phys. 67 (2004) 813.

[2] L.V. Keldysh, Sov. Phys. JETP 20 (1965) 1307.

[3] G.F. Gribakin, M. Yu Kuchiev, Phys. Rev. A 55 (1997) 3760.

[4] L.D. Landau, E.M. Lifshitz, Quantum Mechanics. Non-relativistic Theory, Pergamon, Oxford, 1965.

[5] K. Mishima, M. Hayashi, J. Yi, S.H. Lin, H.L. Selzle, E.W. Schlag, Phys. Rev. A 66 (2002) 033401.

[6] K. Nagaya, K. Mishima, M. Hayashi, S.H. Lin, J. Chem. Phys. 124 (2006) 144303.

[7] A. Scrinzi, M. Geisser, T. Brabec, Phys. Rev. Lett. 83 (1999) 706.

[8] T. Walsh, F.A. Ilkov, J.E. Decker, S.L. Chin, J. Phys. B27 (1994) 3767.

[9] S.L. Chin, Y. Liang, J.E. Decker, F.A. Ilkov, M.V. Ammosov, J. Phys. B25 (1992) L249.

[10] T.W. Gamelin, Complex Analysis, Springer-Verlag New York Inc., 2001. 